\title{
Hot prominence detected in the core of a coronal mass ejection: Analysis of SOHO/UVCS L $\alpha$ and SOHO/LASCO visible-light observations
}

\author{
P. Heinzel ${ }^{1,4}$, R. Susino ${ }^{2}$, S. Jejčič ${ }^{3,1}$, A. Bemporad ${ }^{2}$, and U. Anzer ${ }^{4}$ \\ 1 Astronomical Institute, The Czech Academy of Sciences, 25165 Ondřejov, Czech Republic \\ e-mail: pheinzel@asu.cas.cz \\ 2 INAF-Turin Astrophysical Observatory, 10025 Pino Torinese (TO), Italy \\ 3 Faculty of Mathematics and Physics, University of Ljubljana, 1000 Ljubljana, Slovenia \\ ${ }^{4}$ Max-Planck-Institut für Astrophysik, Karl-Schwarzschild-Str. 1, 85740 Garching bei München, Germany
}

Received 22 September 2015 / Accepted 8 March 2016

\begin{abstract}
Context. The paper deals with the physics of erupting prominences in the core of coronal mass ejections (CME).

Aims. We determine the physical parameters of an erupting prominence embedded in the core of a CME using SOHO/UVCS hydrogen $\mathrm{L} \alpha$ and $\mathrm{L} \beta$ lines and SOHO/LASCO visible light observations. In particular we analyze the CME event observed on August 2, 2000. We develop the non-LTE (NLTE; i.e. considering departures from the local thermodynamic equilibrium - LTE) spectral diagnostics based on $\mathrm{L} \alpha$ and visible light observations.

Methods. Our method is based on 1D NLTE modeling of eruptive prominences and takes into account the effect of large flow velocities, which reach up to $300 \mathrm{~km} \mathrm{~s}^{-1}$ for the studied event (the so-called Doppler dimming). The NLTE radiative-transfer method can be used for both optically thin and thick prominence structures. We combine spectroscopic UVCS observations of an erupting prominence in the core of a CME with visible light images from LASCO-C2 in order to derive the geometrical parameters like projected thickness and velocity, together with the effective temperature and column density of electrons. These are then used to constrain our NLTE radiative transfer modeling which provides the kinetic temperature, microturbulent velocity, gas pressure, ionization degree, the line opacities, and the prominence effective thickness (geometrical filling factor).

Results. Analysis was made for 69 observational points (spatial pixels) inside the whole erupting prominence. Roughly one-half of them show a non-negligible L $\alpha$ optical thickness for flow velocity $300 \mathrm{~km} \mathrm{~s}^{-1}$ and about one-third for flow velocity $150 \mathrm{~km} \mathrm{~s}{ }^{-1}$. All pixels with $\operatorname{L} \alpha \tau_{0} \leq 0.3$ have been considered for further analysis, which is presented in the form of statistical distributions (histograms) of various physical quantities such as the kinetic temperature, gas pressure, and electron density for two representative flow velocities (150 and $300 \mathrm{~km} \mathrm{~s}^{-1}$ ) and non-zero microturbulence. For two pixels co-temporal LASCO visible-light data are also available, which further constrains the diagnostics of the electron density and effective thickness. Detailed NLTE modeling is presented for various sets of input parameters.

Conclusions. The studied CME event shows that the erupting prominence expands to large volumes, meaning that it is a low-pressure structure with low electron densities and high temperatures. This analysis provides a basis for future diagnostics using the METIS coronagraph on board the Solar Orbiter mission.
\end{abstract}

Key words. Sun: filaments, prominences - Sun: coronal mass ejections - Sun: UV radiation - radiative transfer

\section{Introduction}

Prominences are cool and dense objects of the solar atmosphere plunged into the hotter and more rarified solar corona. They appear as bright structures when observed beyond the solar limb and as dark filamentary structures when seen against the solar disk. It is quite usual to classify prominences as quiescent or active prominences based on their lifetimes, location, and physical properties. Quiescent prominences are long-lived structures generally lying along the inversion polarity line or between weak active regions. Although appearing stable for several days (or even weeks), they are highly dynamic objects, subject to flows or small amplitude oscillations as revealed by groundbased and space observations. It is quite accepted now that their support against gravity and thermal insulation from the milliondegree corona are due to small-scale and large-scale magnetic fields (see, e.g., Vial \& Engvold 2015, for a review). Active prominences are located within active regions (AR), adjacent to sunspots, and are relatively short-lived: they are subject to sudden eruptions resulting in lifetimes of several minutes to a few hours. During the eruption, prominences are rapidly accelerated upward to velocities ranging from 100 to $1000 \mathrm{~km} \mathrm{~s}^{-1}$ or more; erupting prominences can be accompanied by solar flares and by the expulsion of surrounding coronal material, triggering coronal mass ejections (CMEs). The subject of solar prominences and CMEs is covered in the book edited by Vial \& Engvold (2015) and in the proceedings from IAUS300 (Schmieder et al. 2013). Information about thermodynamic properties of the prominence plasma can be obtained from the analysis of prominence spectra or high-resolution monochromatic images. Spectroscopic data allow for the determination of several plasma parameters such as kinetic temperature, density, gas pressure, bulk velocity, and ionization state. These quantities are all obtained from the observed spectra using the methods of non-LTE (NLTE; i.e. considering departures from the local thermodynamic equilibrium - LTE) modeling (Labrosse et al. 2010; Heinzel 2015; Labrosse 2015).

The prominence emission is observable in the broad spectral range and consists of several lines formed at temperatures 

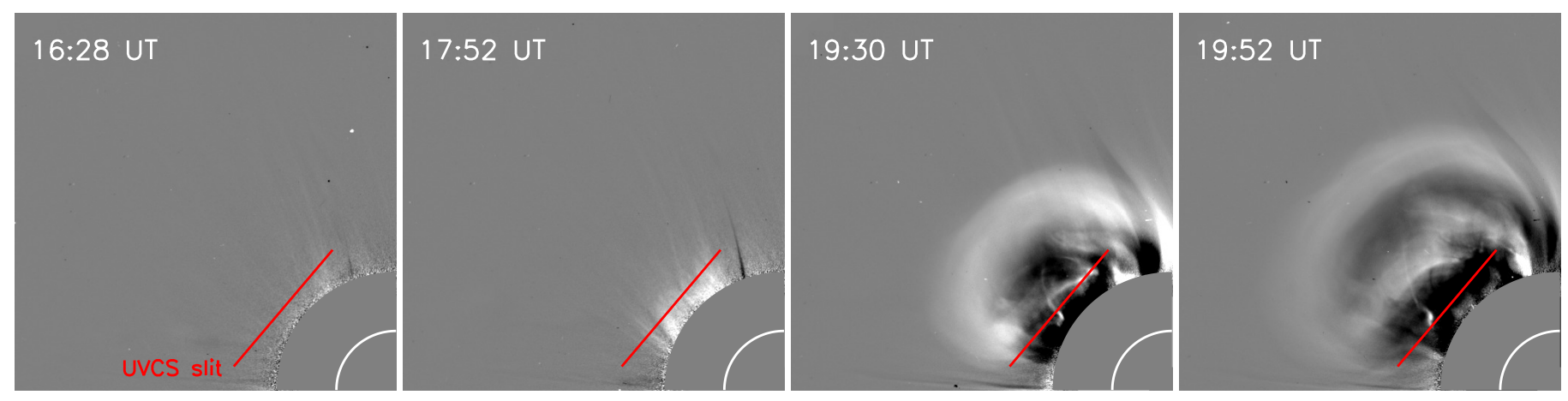

Fig. 1. Sequence of LASCO-C2 base-difference images (frame at 16:04 UT subtracted) of the CME event that occurred on August 2, 2000. The erupting prominence associated with the CME can be recognized in the last two frames on the right. The UVCS slit, centered at $2.3 R_{\odot}$ at a latitude of $40^{\circ} \mathrm{N}$, is superimposed onto the images as a red line for reference.

ranging from chromospheric to coronal values. For instance, the dominant $\mathrm{H} \alpha(\lambda 6562.81 \AA)$ and $\mathrm{L} \alpha(\lambda 1215.67 \AA)$ lines of hydrogen are thought to emerge from the cool prominence core at electron temperatures of about $10^{4} \mathrm{~K}$ and originate in the regime of optically thick plasma. Typical electron densities of the cool core plasma as derived from SOHO/SUMER spectra in quiescent prominences and are on the order of $10^{10}-10^{11} \mathrm{~cm}^{-3}$, while gas pressures are on the order of $0.1-1 \mathrm{dyn}^{-2}$ (e.g., Labrosse et al. 2010). Conversely, the external layers of the prominence, the so-called prominence-corona transition region (PCTR), where the temperature increases beyond $10^{5} \mathrm{~K}$ and the density decreases to values as low as $\sim 10^{8} \mathrm{~cm}^{-3}$, are responsible for optically thin line emission at intermediate temperatures (e.g., Parenti 2015).

In recent decades, the UV coronagraph spectrometer (UVCS) on board SOHO (Kohl et al. 1995) has observed several erupting prominences in the core of CMEs in the form of long, thin filaments that are brighter in lines from the low-ionization species (Kohl et al. 2006). The cooler (and brighter) lines, such as those of $\mathrm{H} \mathrm{I}$ and C III $\left(\sim 5 \times 10^{4} \mathrm{~K}\right)$, are found in narrower cores within more diffuse envelope structures seen in $\mathrm{O}$ VI $\left(\sim 10^{5} \mathrm{~K}\right)$. Ciaravella et al. (2000), for instance, derived an upper limit to the kinetic temperature as low as $10^{4.8} \mathrm{~K}$ from the widths of the Lyman lines in the prominence core of a CME. The presence of ions ranging from $\mathrm{C}^{+}$to $\mathrm{O}^{5+}$ in UVCS spectra, however, suggests a range of temperatures from $\sim 10^{4} \mathrm{~K}$ (or even lower) to $\sim 10^{5} \mathrm{~K}$ (or even higher).

Spectroscopic data can be supplemented by taking advantage of visible light (VL) coronagraphic images, which detect the radiation scattered on free electrons through Thomson scattering. Single-viewpoint VL images, such as those from the LASCO coronagraphs on board SOHO (Brueckner et al. 1995), can be used to evaluate the prominence geometrical structure and plane-of-the-sky (POS) dynamics, and to provide an alternative way to estimate the prominence density and mass (see, e.g., Vourlidas et al. 2000).

Measuring all of these quantities is essential in order to depict the physical state of the prominence and also for the development and testing of models describing the prominence internal structure. Owing to the particular conditions of high density and low temperature, in fact, the interpretation of the prominence observations requires sophisticated models of the prominence plasma in the magnetic field and NLTE radiative transfer computations (Gunár 2014; Heinzel 2015).

In this work, we combine spectroscopic UVCS observations of an erupting prominence in the core of a CME with VL images from LASCO-C2 in order to derive the geometrical parameters
Table 1. Lines identified in UVCS data.

\begin{tabular}{lllc}
\hline \hline $\begin{array}{l}\lambda_{\text {rest }}{ }^{a} \\
(\AA)\end{array}$ & Ion & Transition & $\begin{array}{c}\log T_{\text {form }} \\
(\mathrm{K})\end{array}$ \\
\hline 977.02 & C III & $2 \mathrm{~s}^{2}{ }^{1} \mathrm{~S}_{0}-2 \mathrm{~s} 2 \mathrm{p}{ }^{1} \mathrm{P}_{1}$ & 4.9 \\
1025.72 & H I & $\mathrm{L} \beta$ & 4.5 \\
1031.91 & O VI & $1 \mathrm{~s}^{2} 2 \mathrm{~s}^{2} \mathrm{~S}_{1 / 2}-1 \mathrm{~s}^{2} 2 \mathrm{p}^{2} \mathrm{P}_{3 / 2}$ & 5.5 \\
1036.34 & C II & $2 \mathrm{~s}^{2} 2 \mathrm{p}^{2} \mathrm{P}_{1 / 2}-2 \mathrm{~s} 2 \mathrm{p}^{2}{ }^{2} \mathrm{~S}_{1 / 2}$ & 4.6 \\
1037.02 & C II & $2 \mathrm{~s}^{2} 2 \mathrm{p}^{2} \mathrm{P}_{3 / 2}-2 \mathrm{~s} 2 \mathrm{p}^{2}{ }^{2} \mathrm{~S}_{1 / 2}$ & 4.6 \\
1037.61 & O VI & $1 \mathrm{~s}^{2} 2 \mathrm{~s}^{2} \mathrm{~S}_{1 / 2}-1 \mathrm{~s}^{2} 2 \mathrm{p}^{2} \mathrm{P}_{1 / 2}$ & 5.5 \\
1215.67 & H I & L $\alpha$ & 4.5 \\
\hline
\end{tabular}

Notes. ${ }^{(a)}$ Rest wavelength of the transition.

such as projected thickness and velocity, together with the effective temperature and column density of electrons. These are then used to constrain our NLTE radiative transfer modeling which provides the kinetic temperature, microturbulent velocity, gas pressure, ionization degree, the line opacities, and the prominence effective thickness (geometrical filling factor).

\section{SOHO observations of the CME}

On August 2, 2000 a partial-halo CME left the Sun, propagating in the eastern hemisphere at a latitude of $45^{\circ} \mathrm{N}$ with an initial linear speed of $585 \mathrm{~km} \mathrm{~s}^{-1}$, as reported in the online LASCO CME Catalog (Gopalswamy et al. 2009). The CME was first detected by the LASCO-C2 coronagraph (Brueckner et al. 1995) on board SOHO at 17:52 UT, when the leading edge entered the instrument field of view (FOV) at $2.2 R_{\odot}$. At 19:30 UT, a bright, arch-shaped feature identifiable as the erupting prominence associated with the CME can be recognized in the LASCO-C2 basedifference image located just below the CME front (see Fig. 1).

UVCS observations started on August 2 at 16:52 UT and lasted until 23:47 UT. The UVCS slit, 40 arcmin long and perpendicular to the solar radius, was centered at a latitude of $40^{\circ} \mathrm{N}$ in the eastern quadrant and at a heliocentric distance of $2.3 R_{\odot}$ (see Fig. 1). The slit width was $50 \mu \mathrm{m}$ and the detector mask binned the data over 3 pixels $\left(21^{\prime \prime}\right)$ in the spatial direction. Spectra were acquired in three wavelength intervals: 975.19977.37 $\AA$, 1023.06-1042.30 , and 1211.70-1220.85 $\AA$, with a 2-pixel spectral binning corresponding to a spectral resolution of $0.1983 \AA$ in the first two ranges and $0.183 \AA$ in the third. Table 1 lists the main lines that have been detected, together with the temperature of formation $\left(T_{\text {form }}\right)$ of the emitting ion according 

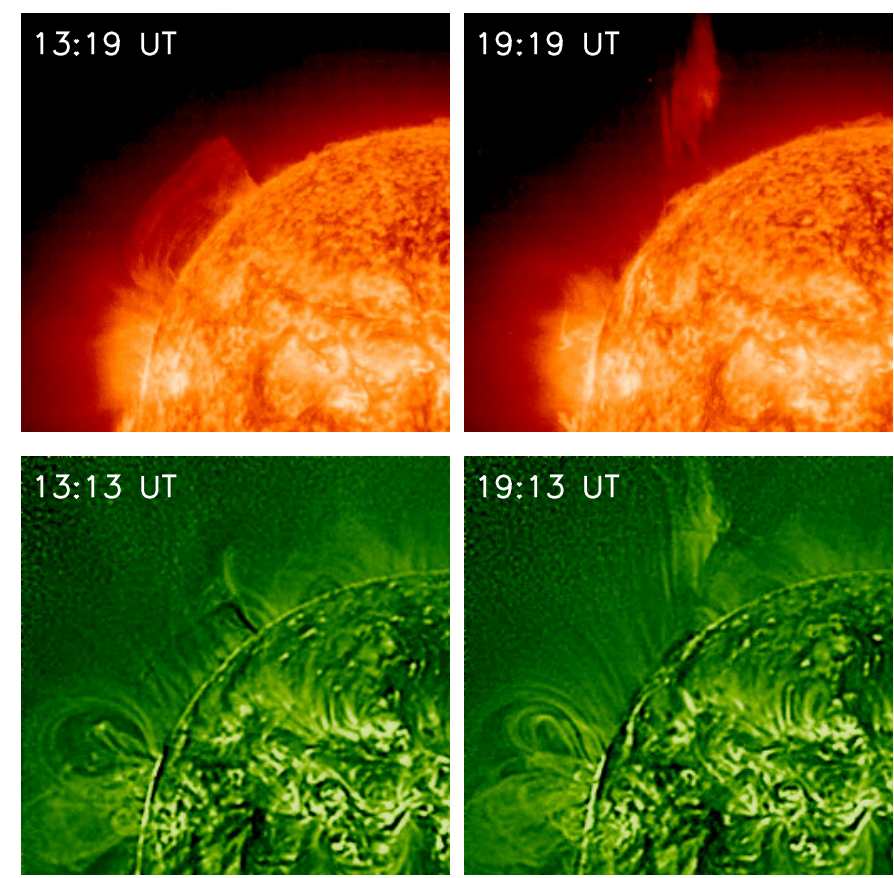

Fig. 2. Prominence as seen before (left panels) and during (right panels) the eruption in the He II $\lambda 304 \AA$ (top panels) and Fe XII $\lambda 195 \AA$ (bottom panels) channels of EIT. The Fe XII $\lambda 195 \AA$ A images have been processed with wavelets in order to highlight faint structures and emission gradients.

to the ionization balance of Bryans et al. (2006). Standard calibration of the UVCS data was performed using the most recent calibration routines included with the Data Analysis Software (DAS), version 51.

During the eruption, the CME front and the prominence crossed the UVCS slit almost centrally and the prominence plasma could be fully sampled.

\subsection{Prominence location and dynamics}

The pre-eruption prominence is visible in images acquired by the Extreme UV Imaging Telescope (EIT; Delaboudinière et al. 1995) as an elongated structure located over the eastern limb of the Sun (see Fig. 2); it appears as a bright emitting feature in the He II $\lambda 304 \AA$ line $\left(\log T_{\text {form }} \approx 4.9\right)$ and as a dark absorbing feature in the Fe XII $\lambda 195 \AA$ line $\left(\log T_{\text {form }} \approx 6.2\right)$. Before the eruption, the southern leg of the prominence was anchored to the solar surface in a region located in the visible part of the disk, while the northern leg was anchored behind the limb. At approximately 16:00 UT, the equilibrium that sustained the prominence is lost and the eruption begins; later on, the EIT $304 \AA$ image at 19:19 UT shows that the northern leg of the prominence is still tied up to the solar surface while the southern leg has been ejected higher up in the corona. This is particularly evident from the composite image reported in Fig. 3 where we show a rough reconstruction of the prominence geometry.

We used LASCO-C2 images to estimate the component of the prominence velocity projected on the plane of the sky, $V_{\mathrm{POS}}$. We considered the two consecutive images where the prominence can be identified in the C2 FOV, at 19:30 UT and at 19:52 UT (see Fig. 1), and measured the average altitude variation $\langle\Delta h\rangle$ of corresponding prominence features recognizable in both images. The resulting average velocity, $\left\langle V_{\mathrm{POS}}\right\rangle=\langle\Delta h\rangle / \Delta t$

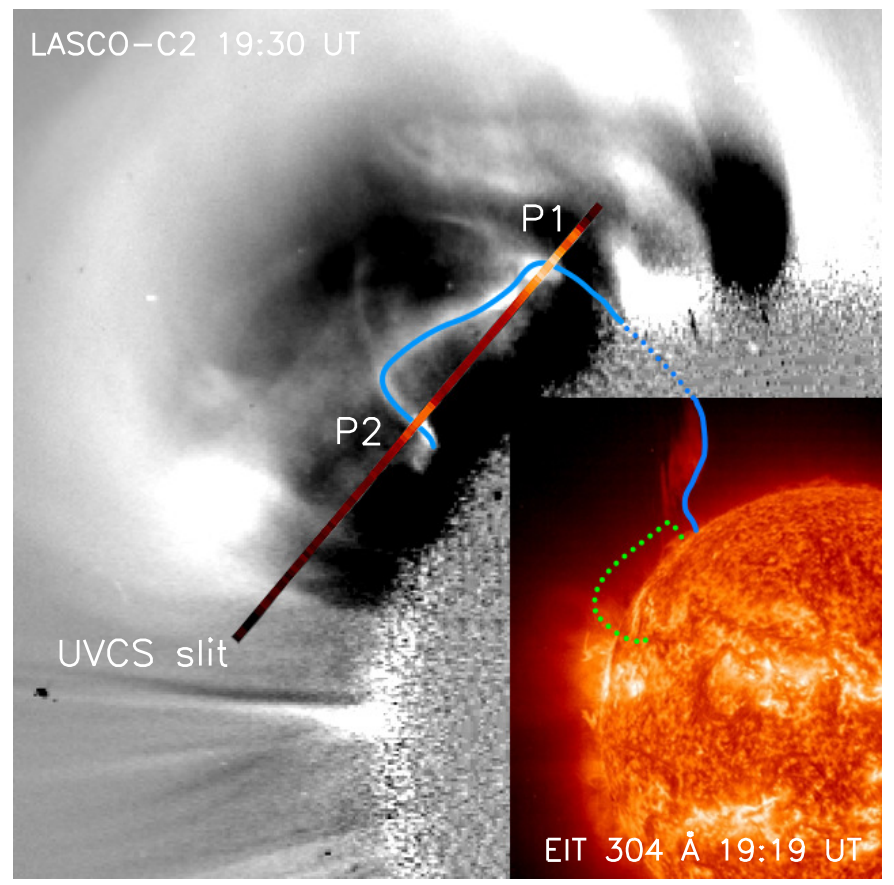

Fig. 3. Composite image of EIT $\lambda 304 \AA$ (19:19 UT), base-difference LASCO-C2 VL (19:30 UT), and intensity distribution of the L $\alpha$ line along the UVCS slit (at 19:30 UT, represented as color gradient). The approximate prominence configurations at the same time (blue line) and before the eruption (green line) are also shown.

$(\Delta t=1334 \mathrm{~s})$, is $280 \pm 30 \mathrm{~km} \mathrm{~s}^{-1}$; the uncertainty arises from a \pm 5 pixel indetermination in locating corresponding features within the prominence. This value is about half of the CME front speed estimated with the same technique from LASCO images. We note, however, that if the prominence was still accelerating during the considered time interval, this average value is most likely an overestimate of the actual POS component of the velocity at 19:30 UT and at the altitude of the UVCS slit, because the prominence has already almost completely crossed the UVCS FOV at that time.

More information on the plasma radial velocity are provided by the analysis of the relative intensities of the $\mathrm{O}$ VI doublet lines at $\lambda \lambda 1032,1037 \AA$. The O VI intensity ratio is 2 for collisional excitation and 4 for resonant scattering at small outward speeds, while at higher speeds it is affected by Doppler dimming (see, e.g., Noci et al. 1987). In most of the bins ( 60\%) within the prominence the ratio $R=I_{1032} / I_{1037}$ is 2 or lower (see Fig. 4). Values of $R \simeq 2$ suggest a radial component of the outflow velocity around or greater than $100 \mathrm{~km} \mathrm{~s}^{-1}$. Nevertheless, in bins where $R<2$, pumping of the O VI $\lambda 1037 \AA$ by the C II $\lambda \lambda 1036$, $1037 \AA$ lines is probably occurring, implying higher radial velocities in the range $\sim 170-370 \mathrm{~km} \mathrm{~s}^{-1}$ (see Raymond \& Ciaravella 2004; Ciaravella et al. 2005). This also indicates that the outflow velocity as inferred from LASCO is probably overestimated. The flow velocities will be further constrained by our modeling presented in Sect. 8.

\section{2. $L \alpha$ spectra}

In what follows, we define two specific points of the prominence, P1 and P2 (see Fig. 3); these are the only two points along the prominence where contemporary and cospatial UVCS and LASCO data are available. Figure 5 shows, as an example, the 


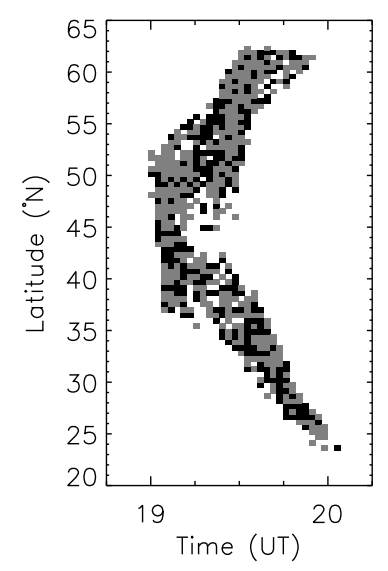

Fig. 4. Intensity ratio of the OVI $\lambda 1032$ and $\lambda 1037$ lines. Grey pixels indicate $R \leq 2$, black pixels $R>2$.
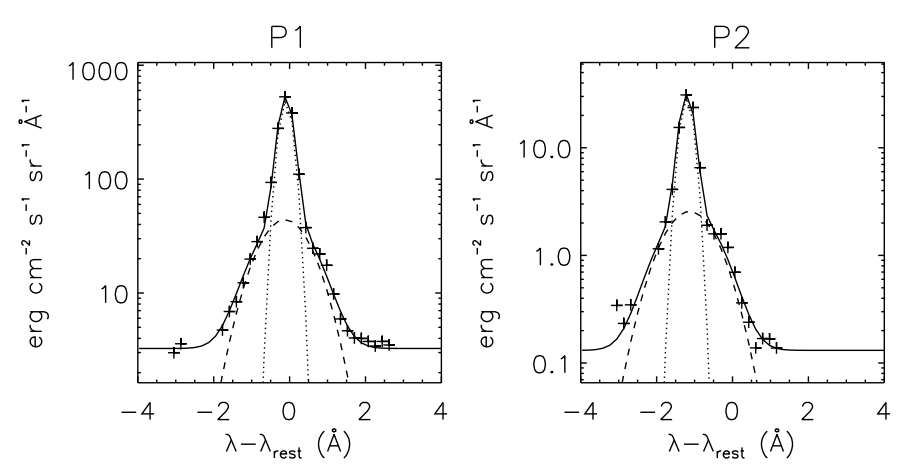

Fig. 5. H I $\operatorname{L} \alpha\left(\lambda_{\text {rest }}=1215.67 \AA\right.$ ) line intensity profiles (crosses) in the two points where the prominence crossed the UVCS slit at 19:30 UT (see Fig. 3). The solid line shows the results of the two-Gaussian fit with a narrow (dotted line) and a broad (dashed line) profiles.

spectral profiles (in log scale) of the $\mathrm{H}$ I L $\alpha$ line in the points $\mathrm{P} 1$ and P2 at 19:30 UT. They appear to be the superposition of two components with different line widths; to check whether these two components were significant in all the data, we made two different attempts by fitting both a single-Gaussian and a doubleGaussian function to the observed profiles in all pixels of the UVCS FOV. A chi-squared analysis (following the method proposed by Kohl et al. 1997) gives evidence that double-Gaussian fits are systematically more reliable and the broad component is not simply an artifact due, for instance, to instrumental broadening effects or contamination by the pre-event corona. We thus derived line intensities, effective temperatures ${ }^{1}$, and Doppler velocities for both the narrow and the broad components.

The most likely interpretation for the two components is that the narrow one represents genuine prominence emission, while the broad one could be due to plasma in the prominence PCTR or alternatively in the hot shroud surrounding the prominence

1 We call "effective" the temperature $T_{\text {eff }}$ that is obtained from the line width $\Delta \lambda_{1 / e}$ using the relationship

$\frac{\Delta \lambda_{1 / e}}{\lambda_{\text {rest }}}=\frac{1}{c} \sqrt{\frac{2 k_{\mathrm{B}} T_{\mathrm{eff}}}{m_{\mathrm{H}}}}=\frac{1}{c} \sqrt{\frac{2 k_{\mathrm{B}} T}{m_{\mathrm{H}}}+\xi^{2}}$,

where $c$ is the speed of light, $k_{\mathrm{B}}$ the Boltzmann constant, $T$ the neutral hydrogen kinetic temperature, $m_{\mathrm{H}}$ the hydrogen mass, and $\xi$ the nonthermal velocity component, which is, in principle, unknown (but see Appendix A).

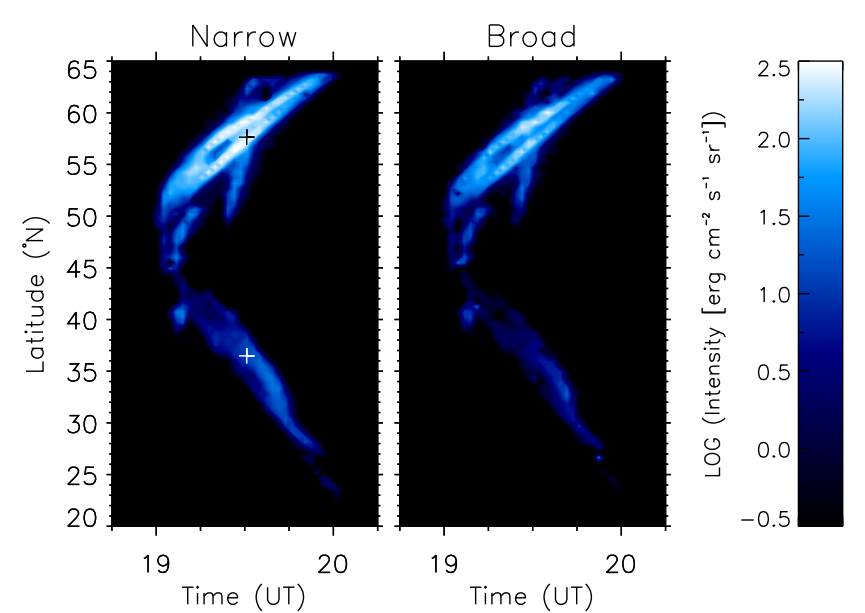

Fig. 6. Intensity distribution of the narrow and broad components of the L $\alpha$ line, plotted with the same color scale as function of time and latitude along the UVCS slit. The crosses in the left panel mark the locations of points $\mathrm{P} 1\left(\sim 57^{\circ} \mathrm{N}\right)$ and $\mathrm{P} 2\left(\sim 37^{\circ} \mathrm{N}\right)$.

(e.g., Habbal et al. 2010) which is dragged by the eruption. In this interpretation, the fact that we can fit the narrow components with Gaussian profiles without any flattening at the line cores seems to indicate that the prominence emission is not optically thick, even if the narrow component is resolved with only five bins with a spectral resolution of $0.183 \AA$ in the $\mathrm{L} \alpha$ wavelength range. For the line-center optical thickness $\tau>1$ it is possible to use the approximate formula

$I(\lambda)=S\left(1-\mathrm{e}^{-\tau}\right)$

where $S=$ const. is the $\mathrm{L} \alpha$ line source function. At $\tau>1$, this expression leads to flat line cores (due to the opacity broadening), which are not recovered in our data sample. Later we obtain realistic values of $\tau$ from our NLTE models and we discuss this issue in greater detail below.

Figure 6 shows the intensity distribution of the two components identified in the L $\alpha$ profiles, as a function of time and latitude along the UVCS slit. The narrow component is more intense than the broad component, on average by a factor of about 2.5. Moreover, the northern leg appears to be significantly brighter than the southern one, by a factor of about 15 , on average. For instance, the intensity of the narrow component is $I \simeq 216 \mathrm{erg} \mathrm{s}^{-1} \mathrm{~cm}^{-2} \mathrm{sr}^{-1}$ at point $\mathrm{P} 1$, while at point $\mathrm{P} 2$ it is only $I \simeq 12 \mathrm{erg} \mathrm{s}^{-1} \mathrm{~cm}^{-2} \mathrm{sr}^{-1}$. This difference could be due to a different plasma density distribution, which can be also related to the different expansion rate of the two legs (a hypothesis suggested by the prominence dynamics evidenced by EIT images), and to a different outflow plasma velocity, which affects the radiative component of the $\mathrm{L} \alpha$ line through the Doppler dimming.

The plasma velocity distribution as derived from the Doppler shift of the line is reported in Fig. 7. The quantity shown in the plots is the line-of-sight component of the plasma velocity, $V_{\mathrm{LOS}}$, as a function of time and latitude along the UVCS slit. As is evident, there is a remarkable difference between the velocity in the two legs of the prominence. Concerning the narrow component, the southern leg is moving towards the observer with an average velocity of $\sim 290 \mathrm{~km} \mathrm{~s}^{-1}$ (for instance, $V_{\mathrm{LOS}} \simeq 297 \mathrm{~km} \mathrm{~s}^{-1}$ at P2). The northern leg, on the contrary, seems only moderately blueshifted, with an average LOS velocity of $\sim 50 \mathrm{~km} \mathrm{~s}^{-1}$ ( $V_{\mathrm{LOS}} \simeq 25 \mathrm{~km} \mathrm{~s}^{-1}$ at $\mathrm{P} 1$ ). The situation is similar in the case of the broad component, indicating that both components are nearly 

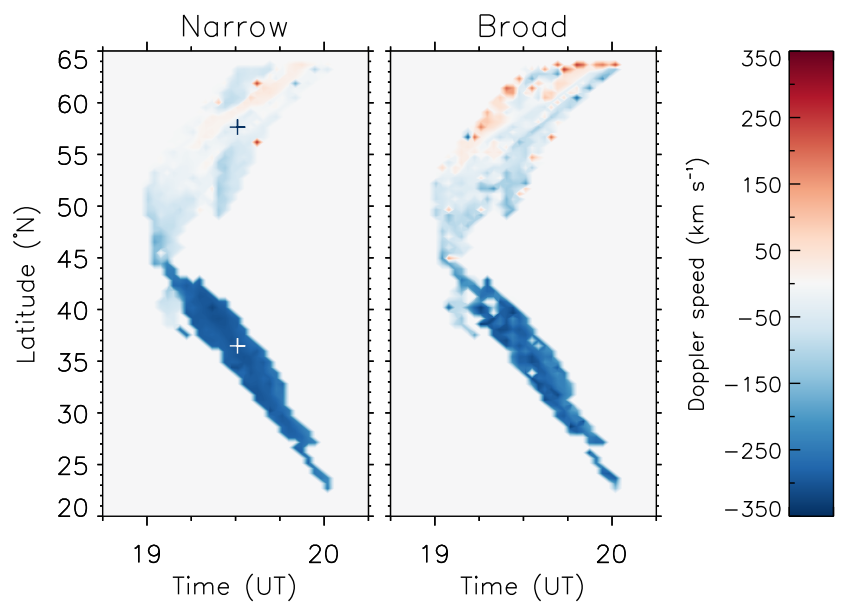

Fig. 7. Same as Fig. 6 for the velocity distribution derived from the Doppler shift of the L $\alpha$ line. As in Fig. 6, the crosses in the left panel indicate the locations of points $\mathrm{P} 1$ and $\mathrm{P} 2$.
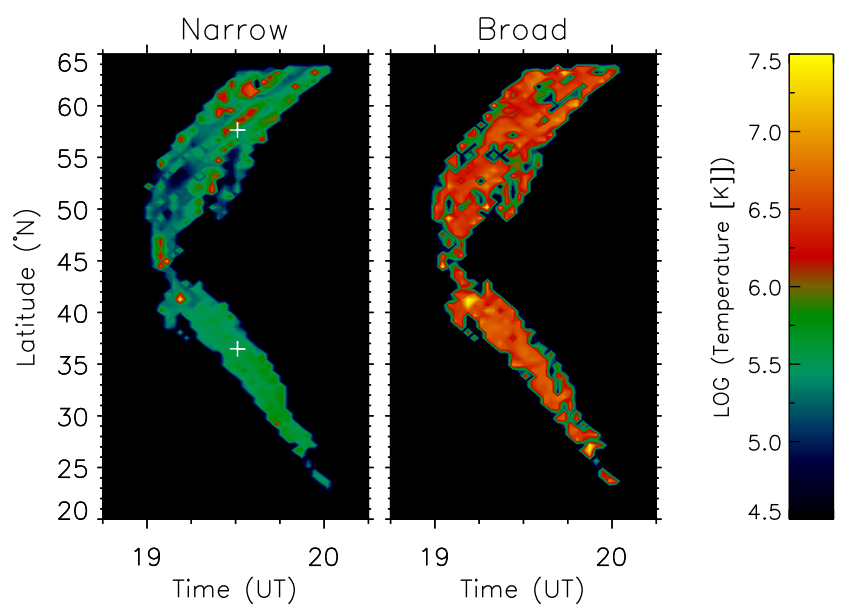

Fig. 8. Effective temperature distribution of the $\mathrm{HI}$ ions in the prominence as derived from the line widths of the two components of the $\mathrm{L} \alpha$ observed profile. As in Fig. 6, the crosses in the left panel indicate the locations of points $\mathrm{P} 1$ and $\mathrm{P} 2$.

co-moving. This result is in good agreement with the scenario depicted by EIT images of the prominence (see Fig. 2) showing that the southern leg is rapidly ejected in the corona during the eruption, unlike the northern one. The difference in the $V_{\mathrm{LOS}}$ values of the two legs may suggest that the prominence is rotating counter-clockwise (as seen from above the source region), but it is also plausible that Doppler shifts are just the line-of-sight component of the prominence expansion.

\section{Range of temperatures from $\mathrm{L} \alpha$ profiles}

Figure 8 shows the effective temperature distribution of prominence plasma derived from the line widths of the two components of the $\mathrm{L} \alpha$ profile. As usual, the effective temperature has been calculated from the line width after deconvolution of the UVCS instrumental profile, which has $F W H M=0.215 \AA$ (Kohl et al. 1999). The narrow component corresponds to much lower temperatures $\left(1.5 \times 10^{5} \mathrm{~K}\right.$ on average) than the broad component (3.5 MK on average). For this reason, as mentioned before, we assume that the former originates from the prominence plasma, which is cooler than the surrounding coronal plasma.
We note, however, that typical kinetic temperatures in standard prominences are on the order of $10^{4} \mathrm{~K}$ (see, e.g., Labrosse et al. 2010), i.e., about one order of magnitude lower. This difference could be partially interpreted as the signature of significant microturbulent (non-thermal) motions within the prominence that lead to broader emergent profiles, hence to larger effective temperatures. For instance, at the wavelength of the $\mathrm{L} \alpha$ transition, non-thermal speeds on the order of $30 \mathrm{~km} \mathrm{~s}^{-1}$ would result in $\sim 50000 \mathrm{~K}$ higher effective temperatures, but even with such high microturbulence we cannot explain the effective temperatures on the order of or higher than $10^{5} \mathrm{~K}$ for which some specific heating is required.

The broad component could come from the prominence-tocorona transition region (PCTR) where high temperatures have been detected by Parenti et al. (2012) or the recently observed prominence hot shrouds (Habbal et al. 2010).

\section{Range of electron densities from SOHO/LASCO $\mathrm{VL}$ images}

Visible light observations provide an independent diagnostic of the electron density. Visible light emission originates from Thomson scattering of photospheric light by free electrons in the solar corona (the so-called K-corona) and by dust in the interplanetary space (the F-corona). Coronagraphic images after removal of the F-corona contamination provide a measure of the total brightness in the band of observation that is given by

$B \simeq K_{\mathrm{T}} \int_{-\infty}^{\infty} n_{\mathrm{e}} \mathrm{d} x=K_{\mathrm{T}} N_{\mathrm{e}}$,

where $K_{\mathrm{T}}$ is a geometrical factor relevant to Thomson scattering, $n_{\mathrm{e}}$ the electron volume density (units of $\mathrm{cm}^{-3}$ ), $x$ the spatial coordinate along the line of sight, and $N_{\mathrm{e}}\left(\mathrm{cm}^{-2}\right)$ the column density, i.e., the total number of electrons per unit area along the LOS.

In order to remove from LASCO images the contribution arising from the F-corona, we follow the method described in Vourlidas et al. (2000). After the coronagraph images are calibrated in units of solar brightness, a suitable pre-event image is subtracted from the frame containing the prominence at 19:30 UT. In this way, the pre-event K-corona is also removed, hence the brightness of each pixel in the backgroundsubtracted image is proportional to the excess number of electrons along the LOS, i.e., to the excess column density. This can be simply obtained from the ratio of the observed brightness $B_{\mathrm{obs}}$ over the brightness $B_{\exp }(\theta)$ of a single electron at some angle $\theta$ along the LOS (usually assumed to be 0 ). The value of $B_{\exp }$ is computed from the Thomson electron scattering function (see Billings et al. 1966) using the SolarSoft routine el theory . pro. The electron column density $N_{\mathrm{e}}$ at points P1 and P2 is $1.48 \times 10^{17}$ and $3.9 \times 10^{16} \mathrm{~cm}^{-2}$, respectively.

The column density can be converted to volume density if the effective thickness of the structure along the LOS, $D_{\text {eff }}$, is known,

$N_{\mathrm{e}}=\int_{-\infty}^{\infty} n_{\mathrm{e}} \mathrm{d} x \simeq\left\langle n_{\mathrm{e}}\right\rangle \cdot D_{\text {eff }}$,

where $\left\langle n_{\mathrm{e}}\right\rangle$ is the average volume density of the structure. The quantity $D_{\text {eff }}$ is unknown and it cannot be simply estimated without some knowledge of the 3D morphology of the structure. In our case, as a first approximation, we assume for the prominence (flux rope) a cylindrical geometry with the LOS thickness equal to the POS projected thickness $D$ that can be estimated directly from the LASCO coronagraphic image. However, 


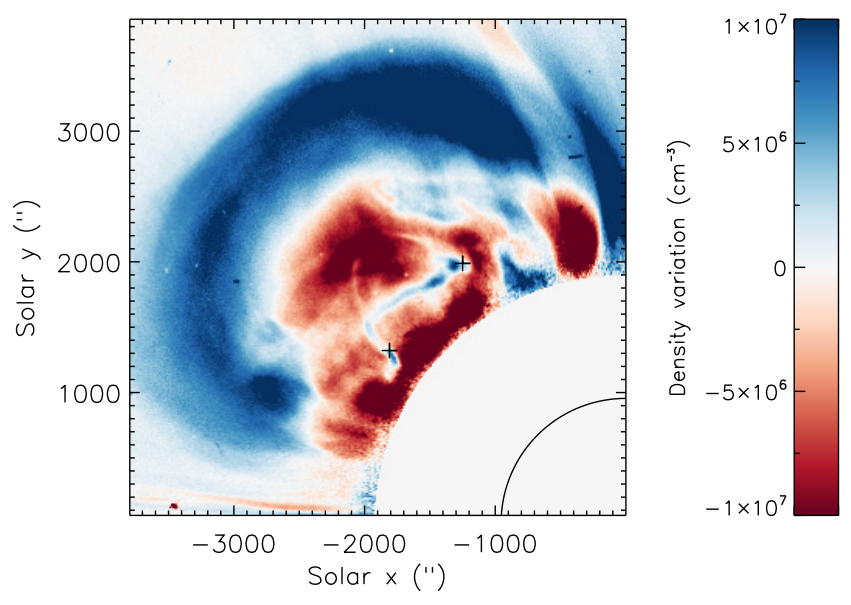

Fig. 9. Electron density distribution derived from the total VL brightness of LASCO-C2 at 19:30 UT. The crosses indicate the positions of points P1 and P2 (see Fig. 3 for comparison). We show the density variations with respect to pre-event corona.

since it is highly probable that the prominence material, when expanding during the eruption, will be highly fragmented (see, e.g., SDO/AIA movies of dark absorbing blobs in the $171 \AA$ and $193 \AA$ channels), it is convenient to introduce a filling factor $f$ to account for such fragmentation:

$D_{\text {eff }}=f \times D$.

Figure 9 shows the electron volume density map derived from the LASCO-C2 image at 19:30 UT, in the simple case of $f=1$ and assuming $D \simeq 6$ pixels $\simeq 5.6 \times 10^{9} \mathrm{~cm}$ as an average lineof-sight prominence thickness. With the $f=1$ assumption these have to be considered as lower-limit values of actual prominence densities. The CME front and the prominence appear as regions with density in excess with respect to the pre-event corona (positive values), while the CME cavity (the red region surrounding the prominence) is depleted (negative values). The average density obtained at P1 and P2 is $2.7 \times 10^{7} \mathrm{~cm}^{-3}$ and $6.8 \times 10^{6} \mathrm{~cm}^{-3}$, respectively. These values are about 3 orders of magnitude lower than densities measured in the core of quiescent prominences $\left(\approx 10^{9}-10^{11} \mathrm{~cm}^{-3}\right.$; see, e.g., Labrosse et al. 2010). This may be the result of a combined effect of the expansion of the prominence during the eruption and a plasma filling factor significantly lower than unity (see below).

\section{5. $\mathrm{L} \alpha$ formation under the hot-prominence conditions}

\subsection{Model description}

In cool quiescent prominences the $\mathrm{L} \alpha$ line source function is dominated by scattering of the incident chromospheric radiation inside the optically thick plasmas (e.g., Heinzel 2015). It was demonstrated by Heinzel et al. (1987) that the hydrogen $\mathrm{L} \alpha$ line formation in quiescent prominences is very sensitive to effects of the partial redistribution (PRD) scattering. In the angleaveraged approximation, this redistribution is a linear combination of the redistribution functions $R_{\mathrm{II}}$ and $R_{\mathrm{III}}$ originally introduced by Hummer (1962). For a detailed description of the PRD theory see Hubeny \& Mihalas (2015). On the other hand, in the hot corona the $\mathrm{L} \alpha$ line is optically thin and the scattering is thus governed by simple Doppler redistribution function $R_{\mathrm{I}}$, which can be taken as angle-dependent (Hummer 1962). In the case of the hot erupting prominences inside the CME analyzed in this paper, we deal with an intermediate situation where some cooler portions could be optically thick while the hotter ones $\left(T \simeq 10^{5} \mathrm{~K}\right)$ become thin in $\mathrm{L} \alpha$. Since we cannot decide a priori whether the plasma is thin at a given location, for each prominence pixel we solve the full NLTE multilevel radiativetransfer problem. For that we use a 1D code based on the Multilevel Accelerated Lambda Iteration (MALI) technique (Rybicki \& Hummer 1991; Heinzel 1995, 2015). The boundary conditions depend on the incident radiation from the surrounding solar disk and we pre-compute the velocity-dependent irradiation for moving structures, which accounts for the effects of Doppler brightening and dimming (the method is described in Heinzel \& Rompolt 1987). We use the five-level hydrogen model atom with continuum and solve the statistical equilibrium for hydrogen excitation and ionization. Unlike the case of cool structures, the $\mathrm{L} \alpha$ intensities of hot prominences will be sensitive to collisional ionization balance. We compared the ionization degree at high temperatures (on the order of $10^{5} \mathrm{~K}$ ) computed by our numerical code with that given in a widely used reference Arnaud $\&$ Rothenflug (1985) and found a reasonable agreement within a few percentage points. Collisional ionization equilibrium consistent with Arnaud \& Rothenflug (1985) was also used in the study by Ciaravella et al. (2003). Moreover, we checked our hydrogen collisional rates against the data provided to us by J. Raymond (priv. comm.). We note that in standard cool prominences the hydrogen is mainly photoionized and its ionization degree largely depends on the incident radiation in the Lyman and Balmer continua (Heinzel 2015).

The NLTE radiative-transfer code is applicable for general optically thick cases, but it doesn't account for details of the angle-dependent redistribution. Angle-averaged PRD leads to results that are similar to the complete redistribution (CRD) because at high temperatures, the scattering takes place mainly within the core of a few Doppler widths where CRD is a good approximation. Also, at relatively high altitudes, the scattering will approximately obey the CRD law, which is exact for a $90 \mathrm{deg}$ scattering angle (function $R_{\mathrm{I}}$ ). For optically thin cases, CRD leads to a Gaussian emission profile, which we also used to fit the observed profiles. Moreover, owing to significant Doppler dimming at large flow velocities, the $\mathrm{L} \alpha$ line source function is more affected by collisions, which lowers the importance of PRD.

\subsection{Incident solar radiation}

The $\mathrm{L} \alpha$ incident radiation used in the MALI code comes from measurements of the solar disk radiation by the LPSP instrument on board the OSO-8 satellite (Gouttebroze et al. 1993). We use the same incident intensity profile as shown by these authors and neglect any center-to-limb variations that are very small. However, it is known that the solar L $\alpha$ brightness varies with time, day by day, and also on the time scale of the solar cycle. Therefore, we used the irradiance data from various resources to determine the actual L $\alpha$ disk intensity at the time of our event. We note that the integrated $\mathrm{L} \alpha$ flux may vary by $80 \%$ between the solar maximum and minimum (e.g., Tobiska et al. 1997) and that our CME observations are made at the maximum of solar activity, while the LPSP profiles were acquired in 1976, i.e., around the solar minimum. The observed frequency-integrated $\mathrm{L} \alpha$ flux at the Earth (irradiance) at the time of our event was estimated to be $F=8.78 \mathrm{erg} \mathrm{s}^{-1} \mathrm{~cm}^{-2}$. Neglecting the center-to-limb variations in $\mathrm{L} \alpha$, we can obtain the specific integrated intensity $I_{\text {tot }}$ using the relation (Hubeny \& Mihalas 2015)

$I_{\mathrm{tot}}=\left(\frac{R}{R_{\odot}}\right)^{2} \frac{F}{\pi}$, 
where $R_{\odot}$ is the solar radius and $R$ the astronomical unit Using the above value of $F$, we obtain $I_{\text {tot }}=1.29 \times$ $10^{5} \mathrm{erg} \mathrm{s}^{-1} \mathrm{~cm}^{-2} \mathrm{sr}^{-1}$. This value is a factor of 1.82 larger than the standard input for the MALI code based on the solar minimum data from OSO-8. We therefore multiply our input $\mathrm{L} \alpha$ profile by this factor in order to simulate the scattering of the $\mathrm{L} \alpha$ radiation in a more consistent way. Ciaravella et al. (2003) mention that the ratio $\mathrm{L} \alpha / \mathrm{L} \beta$ is changing with the cycle, as does $\mathrm{L} \alpha$ itself, but recently Lemaire et al. (2015) demonstrated a similar variations for both the $\mathrm{L} \alpha$ and $\mathrm{L} \beta$ lines based on measurements of the chromospheric radiation during cycle with SOHO/SUMER spectrometer. We thus use their results and multiply our standard $\mathrm{L} \beta$ input by a factor of two.

\section{Model parameters}

We ran the MALI code for 69 pixels. For each latitude along the UVCS slit shown in Fig. 6 we selected the brightest pixel located in correspondence to the maximum observed intensity of the $\mathrm{L} \alpha$ line across the prominence. The input parameters for isothermal-isobaric models are kinetic temperature $T$, gas pressure $p$, effective geometrical thickness $D_{\text {eff }}$, microturbulent velocity $\xi$, flow velocity $V_{\mathrm{f}}$, and the height above the solar surface. In the case of zero microturbulence, the kinetic temperature is equal to effective temperature. For the case of a non-zero microturbulence we use the formula (see Appendix A)

$\xi=7.2 \times 10^{-2} \sqrt{T}$

where

$T=T_{\text {eff }} / 1.31$.

Here $\xi$ is in $\mathrm{km} \mathrm{s}^{-1}$. In this approach the microturbulent velocity increases with the kinetic temperature consistently with observations (e.g., Parenti \& Vial 2007). For our modeling it seems better to assume that $\xi$ is a constant fraction of the sound speed rather than simply a constant over the whole range of temperatures. Assuming a non-zero microturbulence has important consequences. While derived $T_{\text {eff }}$ reproduces well the Doppler broadening of the $\mathrm{L} \alpha$ line, $T$ is the kinetic temperature that enters the evaluation of atomic rates in the NLTE modeling. Moreover, for $T<T_{\text {eff }}$ the hydrogen is less ionized, which leads to larger L $\alpha$ opacities. We actually see that the models with lower temperatures become optically thick in the $\mathrm{L} \alpha$ line. The reason why we introduce the microturbulence is that in such violent events like a CME, we expect rather significant non-thermal motions and their simplest representation is a microturbulence, which can be easily incorporated into the line profile calculations. However, a similar line broadening can be also due to an expansion of the prominence (flux tube) during the course of the CME eruption. We made an estimate of the line-of-sight expansion velocity at the time of our observations by dividing the average POS thickness $D=56000 \mathrm{~km}$ by time needed to reach the UVCS slit height with the flow velocity of $300 \mathrm{~km} \mathrm{~s}^{-1}$ (assuming that the initial prominence thickness is much smaller than $D$ ). This gives an upper-limit estimate of about $18 \mathrm{~km} \mathrm{~s}^{-1}$, comparable to the microturbulent velocities estimated in Appendix A. Considering lower flow velocities (see below), it is possible to arrive at expansion velocities comparable to $12 \mathrm{~km} \mathrm{~s}^{-1}$ reconstructed via triangulation by Bemporad (2009). So it might also be possible that the non-thermal broadening is due to an expansion, which is naturally expected within CMEs (see, e.g., models of Pagano et al. 2015). We note, however, that such an expansion cannot explain the broad component, which requires coronal temperatures.
For the geometrical thickness, it could be possible to use the thickness $D$ estimated at each UVCS slit position (pixel) from the $\mathrm{L} \alpha$ extension shown in Fig. 6, multiplying the time width by an average flow velocity. However, this approach gives unrealistically large widths, reaching substantial fractions of the solar radius in many pixels. This occurs because the flux tube is not generally parallel to to the UVCS slit and thus we are actually measuring the vertical extension of some parts of it rather than the true width. For our extensive grid of models described in the next section we considered a unique effective width $D_{\text {eff }}=1000 \mathrm{~km}$, which gives the filling factor of about $2 \%$ (using the LASCO $D=56000 \mathrm{~km}$ ). This $D_{\text {eff }}$ was chosen rather arbitrarily as a representative thickness for which the histograms in Sect. 7.2 are constructed and discussed. However, from detailed modeling for points P1 and P2 (Sect. 8), we get a broad range of $D_{\text {eff }}$. The point is that we can do this only for these two points where the modeling is also constrained by the LASCO data, while all other pixels provide only the $\mathrm{L} \alpha$ line intensity and thus we cannot derive $D_{\text {eff }}$.

The height above the surface is computed from the position of each pixel along the UVCS slit. These heights reach 1.31.4 solar radii, which implies a significant dilution of the incident radiation and, consequently, a relative enhancement of the collisional excitation in the L $\alpha$ transition. Finally, the flow velocity was determined from LOS Doppler shifts of the L $\alpha$ line (Fig. 6) and from POS motions detected by LASCO C2. This macroscopic flow velocity plays a crucial role in evaluation of the radiative boundary conditions for all lines treated with our five-level hydrogen model atom. In particular we account for Doppler dimming in $\mathrm{L} \alpha$ and $\mathrm{L} \beta$ and Doppler brightening in $\mathrm{H} \alpha$, but all other lines are also treated in the same way. For the grid of 69 models we use two representative values of the radial flow velocity, namely 150 and $300 \mathrm{~km} \mathrm{~s}^{-1}$, while in the detailed diagnostics performed in Sect. 8 we also consider some other values.

\section{Results of the NLTE modeling}

With all these input parameters, we determine the gas pressure that leads to the observed integrated $\mathrm{L} \alpha$ intensity, and as a result we also get the electron density. We run the code in the loop, starting with very low pressures and stop when the computed and observed intensities agree within a few percentage points. Since all narrow-component profiles were previously fitted by a Gaussian, we do not fit the line profiles here, but only the integrated line intensities. However, this appears to be a problem. Roughly one-half of the pixels, and specifically those with kinetic temperatures much lower than $10^{5} \mathrm{~K}$, have the $\mathrm{L} \alpha$ optical thickness larger than 0.1 , which we consider to be the limit of optically thin structure (we extend this limit to 0.3 below in order to obtain better statistics for our histograms). At a flow velocity of $300 \mathrm{~km} \mathrm{~s}^{-1}$ about $10 \%$ of the points are significantly thick with the line-center optical thickness between 10 and 100. At a flow velocity of $150 \mathrm{~km} \mathrm{~s}^{-1}$ about $10 \%$ of the points have optical thickness between 2 and 8. Obviously, the computed line profiles for these pixels are flat or even reversed, and this is not observed. Although our observed line profiles have typically only three points in the line core, they do not seem to be reversed. We discuss this problem in the next subsection.

\subsection{Optically thick models}

Running the NLTE code for all pixels we find that almost onehalf of them have the $\mathrm{L} \alpha$ line center optical thickness larger than 0.1 for flow velocity $300 \mathrm{~km} \mathrm{~s}^{-1}$ and about one-third for 


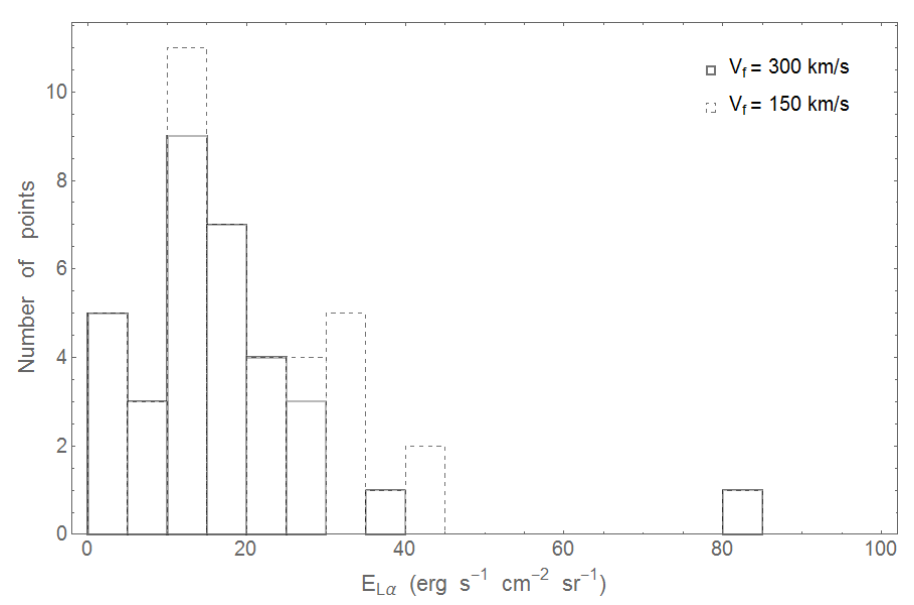

Fig. 10. Distribution of $\mathrm{L} \alpha$ integrated intensities for optically thin structures (four points beyond $E=100$ ), see also Fig. 6. This distribution corresponds to non-zero microturbulence and presents two typical flow velocities of 150 and $300 \mathrm{~km} \mathrm{~s}^{-1}$.

flow velocity $150 \mathrm{~km} \mathrm{~s}^{-1}$. By reducing the flow velocity in the MALI code, the gas pressure has to be reduced in order to get the same energy emitted in the L $\alpha$ line as given by UVCS observations. Below a certain limit of the kinetic temperature, the prominence starts to be optically thick and the convolved profile gives an apparent $T_{\text {eff }}$ that is much higher than the true one. This is even more dramatic for the case of non-zero microturbulence where the kinetic temperature $T$ is lower than $T_{\text {eff }}$. It is $T$ that enters the NLTE code for line opacities, source functions, etc., and for lower temperatures the opacity is higher, which produces the opacity broadening effect. In Fig. 5 we show an example of our Gaussian fit to the UVCS line profiles and we can see that the line core is covered by only three points. This introduces uncertainties in determining the FWHM in cases when the convolved line profile is not exactly Gaussian, i.e., for optically thick situations. The analysis shows that all pixels where we found higher opacities require a more complex study, which we postpone to the next paper. In following sections we discuss our results for pixels that have $\tau_{0} \leq 0.3$. We chose this particular value because it still leads to a realistic estimate of $T_{\text {eff }}$ from the observed line widths (with uncertainty up to about 10\%) and we can use more pixels for our statistics (histograms) shown in the following section.

\subsection{Histograms}

For those pixels that satisfy the condition $\tau_{0} \leq 0.3$ we plot several illustrative histograms showing the statistics of temperature, gas pressure, and electron density for two representative flow velocities $150 \mathrm{~km} \mathrm{~s}^{-1}$ (47 points) and $300 \mathrm{~km} \mathrm{~s}^{-1}$ (37 points), and for non-zero microturbulence. The points were selected from both the northern and southern legs of the prominence (narrow profiles) and they generally correspond to $\mathrm{L} \alpha$ integrated intensities below $100 \mathrm{erg} \mathrm{s}^{-1} \mathrm{~cm}^{-2} \mathrm{sr}^{-1}$. All computed values correspond to $D_{\text {eff }}=1000 \mathrm{~km}$. The estimated geometrical filling factor for the studied pixels is $1.8 \%$ since we take the averaged LASCO value of $56000 \mathrm{~km}$ for geometrical thickness $D$. In Fig. 10 we show the distribution of computed L $\alpha$ intensities, which fit the observed values for flow velocities of 150 and $300 \mathrm{~km} \mathrm{~s}^{-1}$. In all histograms the solid line presents the radial flow velocity $300 \mathrm{~km} \mathrm{~s}^{-1}$ and the dashed line $150 \mathrm{~km} \mathrm{~s}^{-1}$. The reason why we obtain two sets of distributions in Fig. 10 is that

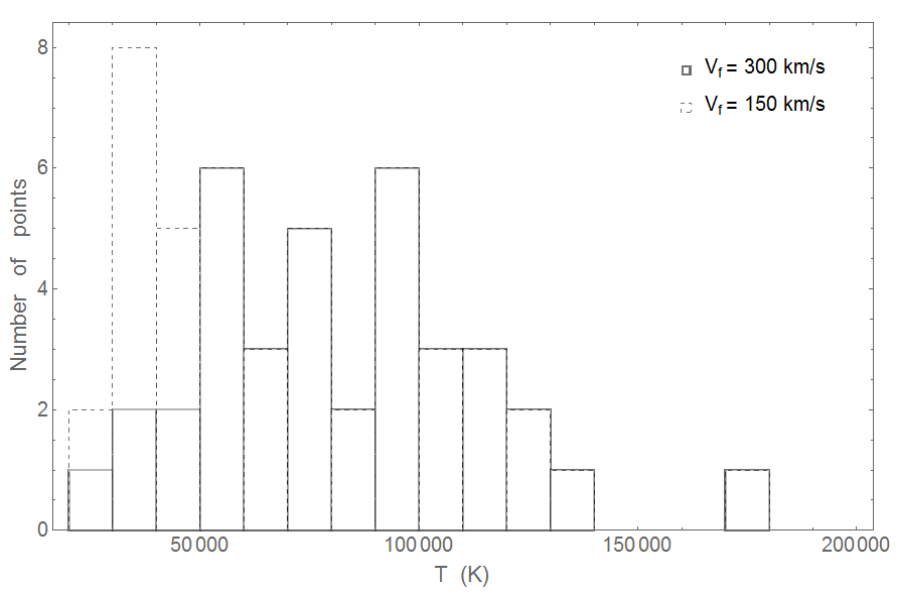

Fig. 11. Kinetic temperature distribution for a flow velocity of 150 and $300 \mathrm{~km} \mathrm{~s}^{-1}$ and non-zero microturbulence.

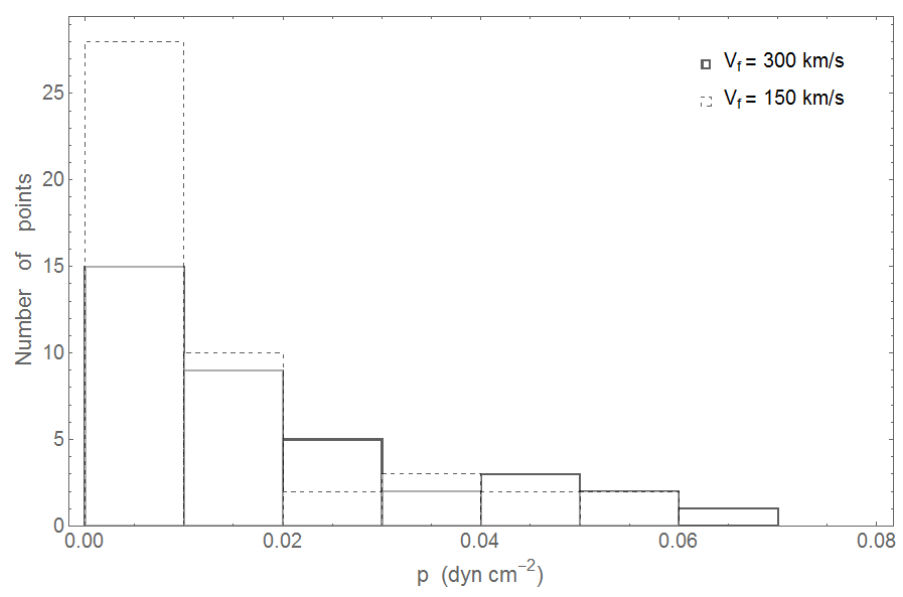

Fig. 12. Gas-pressure distribution for a flow velocity of 150 and $300 \mathrm{~km} \mathrm{~s}^{-1}$ and non-zero microturbulence.

for two different velocities the pixel sample where $\tau_{0} \leq 0.3$ is different. This also applies for other histograms.

The next histogram (Fig. 11) shows the distribution of the kinetic temperature for flow velocity 150 and $300 \mathrm{~km} \mathrm{~s}^{-1}$ and nonzero microturbulence. For the temperature-dependent $\xi$ used in this paper, these distributions are related to corresponding distributions of $T_{\text {eff }}$ by Eq. (7).

The gas pressure derived from the best fit of the $\mathrm{L} \alpha$ line integrated intensities is shown in the next histogram in Fig. 12.

Finally, we show the histogram of the electron densities in Fig. 13. We note that for hot prominence structures, the hydrogen is almost fully ionized and thus we can approximately write

$p=2 n_{\mathrm{e}} k_{\mathrm{B}} T$,

where $k_{\mathrm{B}}$ is the Boltzmann constant. Histograms clearly show that at flow velocity $150 \mathrm{~km} \mathrm{~s}^{-1}$ we get more optically thin pixels because of a reduction in the gas pressure. Lower gas pressure is needed to fit the observed $\mathrm{L} \alpha$ intensity in cases when the Doppler dimming is reduced at lower velocities. According to Eq. (8), the electron density is also reduced. For a given kinetic temperature, the ionization equilibrium implies that the hydrogen ground-state population must also be reduced and this leads to a lowering of the optical thickness. We note that we assume a fixed value of $D_{\text {eff }}=1000 \mathrm{~km}$ for all pixels in the histograms. 
Table 2. Parameters at points P1 and P2 for different NLTE models.

\begin{tabular}{llllllll}
\hline \hline Point & \multicolumn{3}{c}{ P1 } & \multicolumn{3}{c}{ P2 } \\
\hline$T_{\text {eff }}(\mathrm{K})$ & 109400 & 109400 & 70000 & 65000 & 87400 & 87400 & 70000 \\
$T(\mathrm{~K})$ & 83100 & 83100 & 53400 & 49600 & 66700 & 66700 & 53400 \\
$\xi\left(\mathrm{km} \mathrm{s}^{-1}\right)$ & 20.8 & 20.8 & 16.6 & 16.0 & 18.6 & 18.6 & 16.7 \\
$V_{\mathrm{f}}\left(\mathrm{km} \mathrm{s}^{-1}\right)$ & 300 & 100 & 150 & 155 & 300 & 210 & 260 \\
$p\left(\mathrm{dyn} \mathrm{cm}^{-2}\right)$ & 0.068 & 0.028 & 0.0027 & 0.0006 & 0.0068 & 0.0023 & 0.0013 \\
$n_{\mathrm{e}}\left(10^{8} \mathrm{~cm}^{-3}\right)$ & 28 & 12 & 1.7 & 0.4 & 3.5 & 1.2 & 0.86 \\
$D_{\mathrm{eff}}(\mathrm{km})$ & 530 & 1250 & 8500 & 37400 & 1100 & 3340 & 4500 \\
$D(\mathrm{LASCO})(\mathrm{km})$ & 56000 & 56000 & 56000 & 56000 & 56000 & 56000 & 56000 \\
$f(\%)$ & 1 & 2 & 15 & 67 & 2 & 6 & 8 \\
$\left.N_{\mathrm{e}}\left(10^{17} \mathrm{~cm}\right)^{-2}\right)$ & 1.47 & 1.50 & 1.44 & 1.49 & 0.39 & 0.40 & 0.39 \\
$N_{\mathrm{e}}(\mathrm{LASCO})\left(10^{17} \mathrm{~cm}^{-2}\right)$ & 1.48 & 1.48 & 1.48 & 1.48 & 0.39 & 0.39 & 0.39 \\
\hline$E_{\mathrm{L} \alpha}(\mathrm{UVCS})\left(\mathrm{erg} \mathrm{s}^{-1} \mathrm{~cm}^{-2} \mathrm{sr}^{-1}\right)$ & 216.0 & 216.0 & 216.0 & 216.0 & 12.0 & 12.0 & 12.0 \\
$E_{\mathrm{L} \alpha}(\mathrm{model})\left(\mathrm{erg} \mathrm{s}^{-1} \mathrm{~cm}^{-2} \mathrm{sr}^{-1}\right)$ & 216.0 & 217.0 & 224.0 & 219.0 & 12.0 & 12.1 & 12.1 \\
$E_{\mathrm{L} \beta}(\mathrm{UVCS})\left(\mathrm{erg} \mathrm{s}^{-1} \mathrm{~cm}^{-2} \mathrm{sr}^{-1}\right)$ & 0.9 & 0.9 & 0.9 & 0.9 & 0.4 & 0.4 & 0.4 \\
$E_{\mathrm{L} \beta}(\mathrm{model})\left(\mathrm{erg} \mathrm{s}^{-1} \mathrm{~cm}^{-2} \mathrm{sr}^{-1}\right)$ & 26.9 & 11.3 & 3.0 & 0.8 & 1.14 & 0.40 & 0.37 \\
$\tau_{0}(\mathrm{~L} \alpha)$ & 0.11 & 0.11 & 0.71 & 1.0 & 0.07 & 0.07 & 0.19 \\
\hline
\end{tabular}

Notes. UVCS integrated intensities of the $\mathrm{L} \alpha$ and $\mathrm{L} \beta$ lines correspond to narrow components. For description of individual columns see the text.

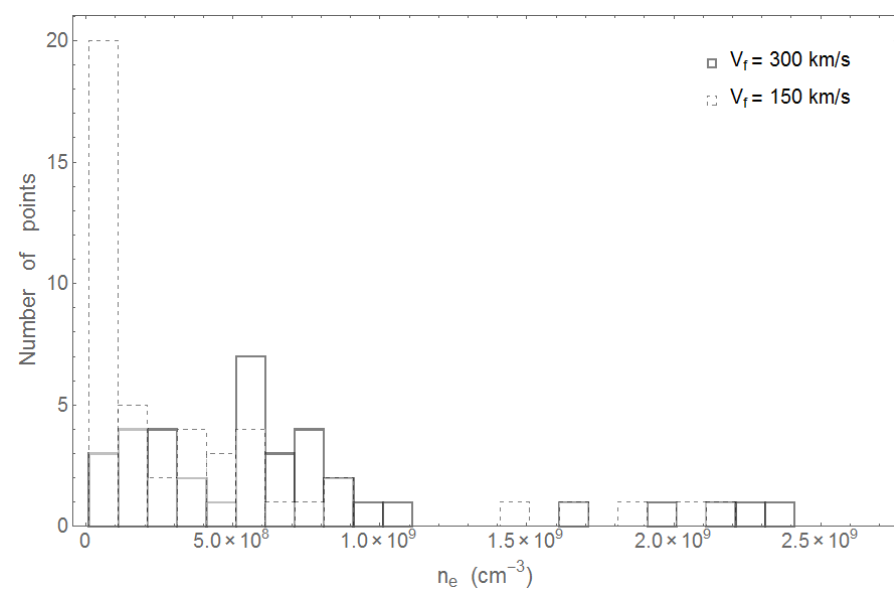

Fig. 13. Electron-density distribution for a flow velocity of 150 and $300 \mathrm{~km} \mathrm{~s}^{-1}$ and non-zero microturbulence.

\section{Electron density and effective thickness diagnostics from UVCS and LASCO}

In this section we propose an iterative method for determination of the mean electron density $n_{\mathrm{e}}$ and geometrical effective thickness $D_{\text {eff }}$ at particular position within the eruptive prominence. We use the MALI code described above and constrain the latter two quantities by the integrated intensity of the $\mathrm{L} \alpha$ line from UVCS (narrow component) and by the column electron density derived from LASCO measurements at points P1 and P2 located at altitudes 975000 and $912000 \mathrm{~km}$, respectively. $n_{\mathrm{e}}$ and $D_{\text {eff }}$ are computed iteratively from estimated starting values. The iterative process is then the following:

as the input we use the observed $E_{\mathrm{L} \alpha}, T, \xi$ (see the Appendix A), $V_{\mathrm{f}}, N_{\mathrm{e}}$ from LASCO and the height above the solar surface. We run the MALI code assuming a starting value of $D_{\text {eff }}^{\text {old }}$ (e.g., $1000 \mathrm{~km}$ ) and search for gas pressure $p$ which will give the computed $\mathrm{L} \alpha$ intensity comparable to the observed one. The resulting mean electron density $n_{\mathrm{e}}^{\text {old }}$ is then used to compute new value of $D_{\text {eff }}$, directly constrained by the LASCO data, i.e.,

$D_{\text {eff }}^{\text {new }}=N_{\mathrm{e}}^{\mathrm{LASCO}} / n_{\mathrm{e}}^{\text {old }}$.
With the next MALI iteration we get $n_{\mathrm{e}}$ which is used to compute new $D_{\text {eff }}$. The convergence was found to be very stable and the final result is independent on the initial guess of $D_{\text {eff }}$, as expected. However, the number of iterations depends on this initial estimate, more iterations are needed if the starting value of the thickness is far from the true one. We note that this iteration method coupled to the MALI code is quite general and can be used for moving structures having any optical thickness in the $\mathrm{L} \alpha$ line. The only free parameter in our NLTE modeling is the microturbulent velocity which we derive using the Appendix A, i.e., is not based on measurements. All other quantities have been derived with certain degree of accuracy from both UVCS and LASCO data (for points P1 and P2).

Using this iterative method, we obtained the set of all relevant parameters for points $\mathrm{P} 1$ and $\mathrm{P} 2$ where simultaneous measurements have been obtained by UVCS and LASCO. Our results are summarized in Table 2. First two columns for each point show solutions using the nominal value of $T_{\text {eff }}$ derived in Sect. 3 from UVCS L $\alpha$ line profiles. In next columns we show examples of solution with lower temperatures. We also varied the flow velocity which may be actually lower than $300 \mathrm{~km} \mathrm{~s}^{-1}$ originally derived from LASCO images (see discussion in Sect. 2.1). $D^{\mathrm{LASCO}}$ is the apparent (POS) geometrical thickness derived from LASCO image and we take a mean value $56000 \mathrm{~km}$ for all studied pixels. At this point we can estimate the geometrical filling factor $f$ also shown in Table 2. Last two lines in the first part of the Table 2 compare the electron column densities from LASCO and from MALI simulation - the differences are very small and the consistent solution was thus achieved. Next part of the Table 2 shows the radiative quantities. Again, very accurate solution was obtained by the iterative procedure for $\mathrm{L} \alpha$ intensity - this is because we search automatically for such gas pressure which gives the computed intensity same as the observed one.

\section{1. $L \beta$ line}

Intensity of the $\mathrm{L} \beta$ line, also observed by UVCS, was not used to constrain the model and we just show the computed values for a comparison with observed ones (narrow component). We varied the temperature and flow velocity to see how they affect 
the $\mathrm{L} \beta$ line intensity. $\mathrm{L} \beta$ from MALI is too high compared to UVCS values assuming the flow velocity $300 \mathrm{~km} \mathrm{~s}^{-1}$. The computed $\mathrm{L} \beta$ intensity decreases with decreasing flow velocity and also with decreasing kinetic temperature - see Table 2. This is consistent with scenario that the actual radial flow velocity is lower than that derived from LASCO images - this is discussed in Sect. 2.1. For point P2 we found a reasonable solution for $\mathrm{L} \beta$ with velocity $210 \mathrm{~km} \mathrm{~s}^{-1}$, keeping the kinetic temperature as derived from measured $T_{\text {eff }}$. However, for point P1 we get solution for somewhat lower velocity around $150 \mathrm{~km} \mathrm{~s}^{-1}$ and lower kinetic temperatures. Such temperatures can be justified by the fact that this pixel becomes optically thick and this causes the opacity broadening of the $\mathrm{L} \alpha$ line from which we originally derived $T_{\text {eff }}$. Therefore, real $T$ must be lower than that obtained from fitting optically-thin Gaussian profiles. Note that we did not use $\mathrm{L} \beta$ for deriving the effective temperature because it is rather noisy and the line-width determination is not accurate enough. For the fourth model of P1 in Table 2, the $\mathrm{L} \alpha$ line is already optically thick and this can explain the need for lower kinetic temperatures. Decreasing the flow velocity, one would expect brighter $\mathrm{L} \beta$ emission because of less important Doppler dimming. This is correct, but because of a decreased dimming, also the $\mathrm{L} \alpha$ intensity would increase unless we keep it fixed in order to stay consistent with the UVCS value. Then $\mathrm{L} \beta$ must decrease as shown in Table 2 .

\section{Conclusions}

In this study we derived physical parameters of a hot prominence plasma detected in the CME core by using SOHO/UVCS hydrogen L $\alpha$ spectra and SOHO/LASCO VL images. The UVCS $\mathrm{L} \alpha$ spectral profiles are fitted with two Gaussians with different broadening, the so-called narrow and broad components. For the prominence we analyzed the narrow component, which has effective temperatures of around $10^{5} \mathrm{~K}$, while the broad one with much higher temperatures $\left(10^{6} \mathrm{~K}\right)$ most probably originates within the PCTR. Both of these components seem to be co-moving in the corona so that the hot one can be considered to be a shroud surrounding the cooler prominence. Gaussian fits seem to indicate that we are dealing with optically thin structures, but we have to bear in mind that we have only three measured points inside the line core. This would not be a problem for the hot (broad) component, but it introduces uncertainties when dealing with cooler plasmas. We selected 69 points (pixels) along the whole prominence and for each point with known position we determine from the L $\alpha$ spectra the effective temperature (after deconvolution with UVCS instrumental profile), Doppler velocity, and integrated L $\alpha$ intensity. With contemporary $\mathrm{SOHO} / \mathrm{LASCO}$ observations at 19:30 UT we estimate the prominence geometrical structure and the prominence flow velocity projected onto the plane of sky. From LASCO data we also get the column electron density at two points P1 and P2.

We developed a diagnostic tool based on the 1D NLTE MALI code, which can be used for both optically thin or thick structures and for large flow velocities on the order of few hundred $\mathrm{km} \mathrm{s}^{-1}$ to determine the physical parameters of a hot prominence plasma. The input parameters are the effective thickness, kinetic temperature, gas pressure, height above the solar surface, flow velocity, and microturbulent velocity. We ran the code in loop with the gas pressure as one free parameter and stopped when the computed integrated $\mathrm{L} \alpha$ intensity is comparable with the observed one within a few percentage points. The code then provides the gas pressure, electron density, integrated L $\alpha$ intensity, and optical thickness $\tau_{0}(\mathrm{~L} \alpha)$. All points having $\tau_{0} \leq 0.3$ are used for our statistics as optically thin pixels. All other points with flat or even reversed computed profiles $\left(\tau_{0}>0.3\right)$ were not studied further in this paper; they generally correspond to cooler and brighter structures.

Results are presented as histograms of the kinetic temperature, gas pressure, and electron density for non-zero microturbulence and for two representative flow velocities 150 and $300 \mathrm{~km} \mathrm{~s}^{-1}$. We introduced the microturbulent broadening of hydrogen spectral lines, and namely of $\mathrm{L} \alpha$, in order to be able to separate the kinetic temperature from the effective value derived directly from the observed line profiles. In our model the microturbulent velocity increases with increasing kinetic temperature as shown in Appendix A. Histograms show that the expanding prominence is hot and has low pressures. This also corresponds to low electron densities. We also found a significant heterogeneity of the erupting prominence with the filling factor between $1 \%$ and $70 \%$. Our results thus constrain the models of CMEs and in particular erupting prominences in their cores. It is interesting to note that our prominence parameters are quite consistent with those obtained from extensive MHD numerical simulations of the CME containing the cool flux rope inside (Pagano et al. 2014). This study also considers the prominence heating by reconnection and plasma compression, leading to the temperatures we observed. Hot prominence plasma is almost fully ionized, but even so there are enough neutral hydrogen atoms to emit the $\mathrm{L} \alpha$ line that was detected by the UVCS coronagraph. This emission is well above the detection limit of the UVCS spectrometer, which is capable of observing much weaker emission from the surrounding corona.

The only pixels where we got simultaneous $\mathrm{L} \alpha$ and visiblelight data are at points $\mathrm{P} 1$ and $\mathrm{P} 2$. To constrain our diagnostics with both $\mathrm{L} \alpha$ intensity and $N_{\mathrm{e}}$ derived from LASCO VL data, we use an iterative method coupled with MALI modeling as described in Sect. 8. This looks very promising and gives quite consistent results, which are summarized in Table 2 . The method is quite general and works for both optically thin and thick structures. As shown in Table 1, some other lines have also been observed for this particular event and we plan to focus on the analysis of the hydrogen $\mathrm{L} \beta$ line, which will provide some independent diagnostics. Our modeling can be extended to 2D geometry (see Heinzel \& Anzer 2001) in order to better describe the radiation transport inside the long prominence legs (flux rope). However, this concerns mainly the optically thick structures, while our present study was restricted to pixels where $\operatorname{L} \alpha$ was found to be optically thin, which means that the actual geometry does not play a role.

Techniques similar to that developed in this paper will be applicable to derive physical parameters of erupting prominences embedded in the core of CMEs and crossing the instrumental FOV of the future METIS coronagraph on board the Solar Orbiter mission. The advantage of METIS is that we will get cospatial and co-temporal L $\alpha$ and VL images for the whole FOV, while here we were able to rely only on independent statistics from UVCS and LASCO with the exception of two points where the prominence crossed the UVCS slit at the time of the LASCO observations. On the other hand, METIS will not provide spectroscopic data (line profiles), thus some assumption on the temperature will be required or it will appear as a free parameter in the NLTE modeling.

Acknowledgements. P.H. and S.J. thank the Torino observatory for support and hospitality during their visit. P.H. acknowledges the support from MPA during his stay in Garching and from the University of Ljubljana during his stays in Slovenia. S.J. was partially supported by the Ondrejov observatory. This work 
was funded through the Czech-ESA PRODEX project "Solar Orbiter - METIS", through the grant of the Czech Funding Agency No. 16-18495S and through the project RVO 67985815. The work of R.S. has been funded by the Agenzia Spaziale Italiana through contracts ASI/INAF No. I/013/12/0 and I/013/12/0-1 We thank the referee John Raymond for his constructive comments and suggestions which largely improved the paper.

\section{References}

Arnaud, M., \& Rothenflug, R. 1985, A\&AS, 60, 425

Bemporad, A. 2009, ApJ, 701, 298

Billings, D. E. 1966, A guide to the Solar Carona (New York: Academic Press)

Brueckner, G. E., Howard, R. A., Koomen, M. J., et al. 1995, Sol. Phys., 162, 357

Bryans, P., Badnell, N. R., Gorczyca, T. W., et al. 2006, ApJS, 167, 343

Ciaravella, A., Raymond, J. C., Thompson, B. J., et al. 2000, ApJ, 529, 575

Ciaravella, A., Raymond, J. C., van Ballegooijen, A., et al. 2003, ApJ, 597, 1118

Ciaravella, A., Raymond, J. C., Kahler, S. W., Vourlidas, A., \& Li, J. 2005, ApJ, 621,1121

Delaboudinière, J.-P., Artzner, G. E., Brunaud, J., et al. 1995, Sol. Phys., 162, 291

Engvold, O., Hirayama, T., Leroy, J.-L., Priest, E. R., \& Tandberg-Hanssen, E. 1990, in Dynamics of Quiescent Prominences, eds. V. Ruždjak, \& E Tandberg-Hanssen (Springer-Verlag), Lect. Notes Phys., 363, 294

Gopalswamy, N., Yashiro, S., Michalek, G., et al. 2009, Earth Moon Planets, 104,295

Gouttebroze, P., Heinzel, P., \& Vial, J.-C. 1993, A\&AS, 99, 513

Gunár, S. 2014, in Nature of Prominences and their Role in Space Weather, eds. B. Schmieder, J.-M. Malherbe, \& S. T. Wu (Cambridge Univ. Press), Proc IAU Symp., 300, 59

Habbal, S. R., Druckmuller, M., Morgan, H., et al. 2010, ApJ, 719, 1362

Heinzel, P. 1995, A\&A, 299, 563

Heinzel, P. 2015, in Solar Prominences, eds. J.-C. Vial, \& O. Engvold (Springer), Chap. 5

Heinzel, P., \& Anzer, U. 2001, A\&A, 375, 1082

Heinzel, P., \& Rompolt, B. 1987, Sol. Phys., 110, 171

Heinzel, P., Gouttebroze, P., \& Vial, J.-C. 1987, A\&A, 183, 351

Hubeny, I., \& Mihalas, D. 2015, Theory of Stellar Atmospheres (Princeton: Princeton Univ. Press)

Hummer, D. 1962, MNRAS, 125, 21

Kohl, J. L., Esser, R., Gardner, L. D., et al. 1995, Sol. Phys., 162, 313

Kohl, J. L., Noci, G., Antonucci, E., et al. 1997, Sol. Phys., 175, 613

Kohl, J. L., Esser, R., Cranmer, S. R., et al. 1999, ApJ, 510, 59

Kohl, J. L., Noci, G., Cranmer, S., \& Raymond, J. C. 2006, A\&ARv, 13, 31

Labrosse, N. 2015, in Solar Prominences, eds. J. C. Vial, \& O. Engvold (Springer), Chap. 6

Labrosse, N., Heinzel, P., Vial, J.-C., et al. 2010, Space Sci. Rev., 151, 243

Lemaire, P., Vial, J. C., Curdt, W., Schühle, U., \& Wilhelm, K. 2015, A\&A, 581, A26

Noci, G., Kohl, J. L., \& Withbroe, G. L. 1987, ApJ, 315, 706

Pagano, P., Mackay, D. H., \& Poedts, S. 2014, A\&A, 568, A120

Parenti, S. 2015, in Solar Prominences, eds. J.-C. Vial, \& O. Engvold (Springer), Chap. 3

Parenti, S., \& Vial, J.-C. 2007, A\&A, 469, 1109

Parenti, S., Schmieder, B., Heinzel, P., \& Golub, L. 2012, ApJ, 754, 66

Raymond, J. C., \& Ciaravella, A. 2004, ApJ, 606, L159

Rybicki, G. B., \& Hummer, D. G. 1991, A\&A, 245, 171

Schmieder, B., Malherbe, J.-M., \& Wu, S. T. 2013, Nature of Prominences and their Role in Space Weather (Cambridge Univ. Press), Proc. IAU Symp., 300

Tobiska, W. K., Pryor, W. R., \& Ajello, J. M. 1997, Geophys. Res. Lett., 24, 1123

Vial, J. C., \& Engvold, O. 2015, Physics of Solar Prominences (Springer)

Vourlidas, A., Subramanian, P., Dere, K. P., \& Howard, R. A. 2000, ApJ, 534, 456

\section{Appendix A: Microturbulent velocity}

It is well established that even in the case of quiescent prominences, microturbulence is present (see, e.g., the Hvar Reference Atmosphere of Quiescent Prominences in Engvold et al. 1990) and for violent eruptions like a CME even more turbulent plasmas are expected. Moreover, as demonstrated by Parenti \& Vial (2007), the microturbulent velocity $\xi$ increases with the kinetic temperature. Since we deal here with a wide range of temperatures (see histograms), it is natural to model an increase of the microturbulent velocity $\xi$ with temperature. To achieve this we use the approach of Heinzel \& Anzer (2001), where $\xi$ is expressed as a constant fraction $\epsilon$ of the sound speed $c_{\mathrm{S}}$, which depends on the temperature

$\xi=\epsilon c_{\mathrm{s}}$,

where

$c_{\mathrm{s}}^{2}=\gamma \frac{k_{\mathrm{B}} T}{m}$.

Here $\gamma$ is the ratio of specific heats and $m$ is the mean molecular mass

$m=\frac{1+4 \alpha}{1+\alpha+i} m_{\mathrm{H}}$

where $\alpha$ is the helium abundance relative to hydrogen (we take $\alpha=0.1), i$ is the ionization degree of hydrogen, and $m_{\mathrm{H}}$ is the mass of the hydrogen atom. Since our temperatures peak around $10^{5} \mathrm{~K}$ (see histograms), we simply take $i=1$. For low temperatures (our coolest pixels) $i$ would be around 0.5 , which slightly affects the results. Moreover, in this formula we have neglected the helium ionization, which also has a marginal effect. Using these expressions we get

$\xi=\epsilon \sqrt{\frac{\gamma k_{\mathrm{B}} T}{m}}$

and

$T_{\mathrm{eff}}=T\left(1+\frac{1}{2} \epsilon^{2} \gamma \frac{m_{\mathrm{H}}}{m}\right)$.

In this paper we set $\epsilon=0.5$ and with $\gamma=5 / 3$ and $m=m_{\mathrm{H}} / 1.5$ we finally get

$T=T_{\text {eff }} / 1.31$

and

$\xi=7.2 \times 10^{-2} \sqrt{T}$.

Here $\xi$ is in $\mathrm{km} \mathrm{s}^{-1}$. This temperature dependence of $\xi$ roughly fits the variations obtained by Parenti \& Vial (2007) for quiescent prominences. At temperatures higher than $10^{5} \mathrm{~K}$ our formula gives somewhat larger $\xi$, but this could be attributed to a more violent behavior of the CME. 